EUROPEAN JOURNAL OF PURE AND APPLIED MATHEMATICS

Vol. 13, No. 3, 2020, 427-443

ISSN 1307-5543 - www.ejpam.com

Published by New York Business Global

\title{
Limit points and separation axioms with respect to supra semi-open sets
}

\author{
T. M. Al-shami ${ }^{1, *}$, E. A. Abo-Tabl ${ }^{2,3}$, B. A. Asaad ${ }^{4,5}$, M. A. Arahet ${ }^{6}$ \\ 1 Department of Mathematics, Sana'a University, Sana'a, Yemen \\ 2 Department of Mathematics, College of Arts and Sciences,Methnab, Qassim University, \\ P. O. Box 931, Buridah 51931, Methnab, Saudia Arabia \\ 3 Department of Mathematics, Faculty of Science, Assiut University, Assiut, Egypt \\ 4 Department of Computer Science, College of Science, Cihan University-Duhok, Kurdistan \\ Region, Iraq \\ 5 Department of Mathematics, Faculty of Science, University of Zakho, Kurdistan Region, \\ Iraq \\ 6 Department of Mathematics, Amran University, Amran, Yemen
}

\begin{abstract}
Sometimes we need to minimize the conditions of topology for different reasons such as obtaining more convenient structures to describe some real-life problems, or constructing some counterexamples whom show the interrelations between certain topological concepts, or preserving some properties under fewer conditions of those on topology. To contribute this research area, in this paper, we establish some new concepts on supra topological spaces using supra semi-open sets and give some characterizations of them. First, we introduce a concept of supra semi limit points of a set and study main properties, in particular, on the spaces that possess the difference property. Second, we define and investigate new separation axioms, namely supra semi $T_{i}$-spaces $(i=0,1,2,3,4)$ and give complete descriptions for each one of them. We provide some examples to show the relationships between them as well as with $S T_{i}$-space.
\end{abstract}

2020 Mathematics Subject Classifications: 54A05, 54C10, 54D10, 54D15

Key Words and Phrases: Supra semi-open set, supra semi limit point, $S s T_{i}$-space $(i=$ $0,1,2,3,4)$

\section{Introduction and preliminaries}

A structure on a nonempty set $X$ is a subset of its power set $P(X)$, topological spaces is an example of structure satisfying three conditions. In fact, topological spaces have been generalized in many ways. Alexendroff [12], in 1940, developed abstract spaces where

* Corresponding author.

DOI: https://doi.org/10.29020/nybg.ejpam.v13i3.3743

Email addresses: tareqalshami83@gmail.com (T. M. Al-shami),

a.adotabl@qu.edu.sa (E. A. Abo-Tabl), baravan.asaad@uoz.edu.krd (B. A. Asaad), malroheet@yahoo.com (M. A. Arahet) 
he strengthened the intersection condition. Mashhour et al. [21], in 1983, considered supra topological spaces by neglecting the intersection condition. Maki et al. [20], in 1996, presented a minimal structure as a collection contains the empty and universal sets. Császár [13], in 2002, introduced generalized topological spaces if it contains the empty set and is closed under a nonempty union; and in 2011, he [14] studied a weak structure as a collection contains the empty set. Al-Odhari [1], in 2015, defined infra topological spaces by dropping only the union condition.

Many aspects of such spaces have already been studied. Supra topological spaces is one of the most important developments of general topology in recent years. A family $\mu$ of subsets of a nonempty set $X$ is called a supra topology if it satisfies two conditions: The first one is the empty and universal sets belong to $\mu$; and the second one is it is closed under arbitrary union. Some authors remove the empty set from the first condition of a supra topology because it is obtained from the second condition as the union of an empty collection of sets. Mashhour et al. [21] studied some basic operators, continuity and separation axioms on supra topological spaces. Al-shami [2] investigated the classical topological notions such as limit points of a set, compactness, and separation axioms on the supra topological spaces. He [7] also studied paracompactness on supra topological spaces. It should be noted that the supra topological frame can be more convenient to solve some practical problems and to model some phenomena as pointed out in [19].

Some results via topology do not still valid via supra topology such as the distribution of the closure operator between the union of two sets and the distribution of the interior operator between the intersection of two sets. Also, the property of a compact subset of a $T_{2}$-space is closed is invalid on the supra topologies.

To extend a class of supra open sets, the notion of supra $\alpha$-open [15], supra pre-open [24], supra $b$-open [26], supra $\beta$-open [18], supra $R$-open [16] and supra semi-open sets [3] have been introduced and their main properties have been discussed. These generalizations of supra open sets were defined in a similar way of defining them on general topology. In other words, their definitions were formulated using supra interior and supra closure operators instead of interior and closure operators. These generalizations have been utilized to define new versions of compactness, connectedness and separation axioms, see, for example $[5,6,17,22,25]$. The class of supra $R$-open sets has been studied in $[4,11]$ under the name of somewhere dense sets. Mustafa and Qoqazeh [23] took advantage of supra $D$-sets to define separation axioms on supra topological spaces. Recently, Al-shami and El-Shafei $[9,10]$ have studied separation axioms on supra soft topological spaces and supra soft topological ordered spaces.

The layout of the paper is as following: In Section (2), we explore a concept of supra semi limit points of a set. In Section (3), we initiate new types of separation axioms using supra semi-open sets and illustrate the relationships between them with the help of examples. Section (4) concludes the paper with summary and further works.

In the rest of this section, we mention some definitions and results of supra topology and supra semi-open sets that make this study self-contained and easy to read. 
Definition 1. [21] A family $\mu$ of subsets of a nonempty set $X$ is called a supra topology provided that the following two conditions hold.

(i) $X$ and $\emptyset \in \mu$.

(ii) $\mu$ is closed under arbitrary union.

Then the pair $(X, \mu)$ is called a supra topological space. Every element of $\mu$ is called a supra open set and its complement is called a supra closed set.

Remark 1. (i) $\mu$ is called an associated supra topology with a topology $\tau$ if $\tau \subseteq \mu$.

(ii) Through this paper, we consider $(X, \mu)$ and $(Y, \nu)$ are associated supra topological spaces with the topological spaces $(X, \tau)$ and $(Y, \theta)$, respectively.

Definition 2. [21] Let $A$ be a subset of $(X, \mu)$. Then int ${ }^{\mu}(A)$ is the union of all supra open sets contained in $A$ and $c l^{\mu}(A)$ is the intersection of all supra closed sets containing $A$.

If there is no confusion, we write int $(A)$ and $c l(A)$ in the places of int $t^{\mu}(A)$ and $c l^{\mu}(A)$, respectively.

Definition 3. [3] $A$ subset $A$ of $(X, \mu)$ is said to be supra semi-open if $A \subseteq \operatorname{cl}(\operatorname{int}(A))$.

Definition 4. [3] For a subset $A$ of $(X, \mu)$, sint ${ }^{\mu}(A)$ is the union of all supra semi-open sets contained in $A$ and $\operatorname{scl}^{\mu}(A)$ is the intersection of all supra semi-closed sets containing A.

If there is no confusion, we write $\operatorname{sint}(A)$ and $\operatorname{scl}(A)$ in the places of $\operatorname{sint}^{\mu}(A)$ and $s c l^{\mu}(A)$, respectively.

Definition 5. [3] A map $g:(X, \mu) \rightarrow(Y, \nu)$ is said to be:

(i) supra semi-continuous if the inverse image of each open subset of $Y$ is a supra semiopen subset of $X$.

(ii) supra semi-open (resp. supra semi-closed) if the image of each open (resp. closed) subset of $X$ is a supra semi-open (resp. supra semi-closed) subset of $Y$.

Definition 6. [2] Let $A$ be a subset of $(X, \mu)$. The family $\mu_{A}=\{A \cap G: G \in \mu\}$ is called a supra relative topology on $A$. A pair $\left(A, \mu_{A}\right)$ is called a supra subspace of $(X, \mu)$.

Definition 7. [8] $\beta$ is called a basis for a supra topology $(X, \mu)$ if every member of $\mu$ can be expressed as a union of elements of $\beta$.

Definition 8. [8] Let $\left\{\left(X_{i}, \mu_{i}\right): i=1,2, \ldots, n\right\}$ be the collection of supra topological spaces. Then $\beta=\prod_{i=1}^{n} \mu_{i}=\left\{\prod_{i=1}^{n} G_{i}: G_{i} \in \mu_{i}\right\}$ defines a basis for a supra topology $T$ on $X=\prod_{i=1}^{n} X_{i}$. The pair $(X, T)$ is called a finite product supra spaces.

Proposition 1. [8] Let $A$ and $B$ be two subsets of $(X, \mu)$ and $(Y, \nu)$, respectively. Then:

(i) $\operatorname{cl}(A) \times \operatorname{cl}(B)=\operatorname{cl}(A \times B)$.

(ii) $\operatorname{int}(A) \times \operatorname{int}(B)=\operatorname{int}(A \times B)$. 


\section{Limit points of a set with respect to supra semi-open sets}

In this section, we introduce and study supra semi limit points of a set. We explore many properties of them and discuss their behaviour on the spaces that possess the difference property. For more illustration of the presented findings, some interesting examples are given.

Definition 9. A subset $A$ of $(X, \mu)$ is said to be a supra semi neighbourhood of $x \in X$ provided that there is a supra semi open set $F$ containing $x$ such that $x \in F \subseteq A$.

Definition 10. A point $x \in X$ is said to be a supra semi limit point of a subset $A$ of $(X, \mu)$ provided that every supra semi neighborhood of $x$ contains at least one point of $A$ other than $x$ itself.

All supra semi limit points of $A$ is said to be a supra semi derived set of $A$ and is denoted by $A^{s \prime}$.

Proposition 2. If $A \subseteq B$, then $A^{s \prime} \subseteq B^{s \prime}$ for every $A, B \subseteq(X, \mu)$.

Proof. Straightforward.

Corollary 1. We have the following results for every $A, B \subseteq(X, \mu)$.

(i) $A^{s \prime} \cup B^{s \prime} \subseteq(A \cup B)^{s \prime}$.

(ii) $(A \cap B)^{s \prime} \subseteq A^{s \prime} \cap B^{s \prime}$.

The following example illustrates that the converse of the above proposition and corollary fails.

Example 1. Let $\mu=\{\emptyset, X,\{1,2\},\{2,3\},\{1,2,3\}\}$ be a supra topology on $X=\{1,2,3,4\}$. Then $\{\emptyset, X,\{1,2\},\{2,3\},\{1,2,3\},\{1,2,4\},\{2,3,4\}\}$ is the collection of all supra semiopen subsets of $(X, \mu)$. If $A=\{2\}, B=\{2,4\}, C=\{1,3,4\}$ and $D=\{2,3,4\}$, then $A^{s \prime}=\{1,3,4\}, B^{s \prime}=\{1,3,4\}, C^{s \prime}=\{2,4\}$ and $D^{s \prime}=\{1,3,4\}$. Now, one readily checks the following:

(i) $B^{s \prime} \subseteq A^{s \prime}$, but $B \nsubseteq A$.

(ii) $B^{s \prime} \cup D^{s \prime}=\{1,3,4\}$ and $(B \bigcup D)^{s \prime}=X^{s \prime}=X$.

(iii) $B^{s \prime} \cap C^{s \prime}=\{4\}$ and $(B \cap C)^{s \prime}=\{4\}^{s \prime}=\emptyset$.

Proposition 3. Let $A$ be a subset of $(X, \mu)$ and $x \in X$. Then $x \in A^{s \prime}$ if and only if $x \in(A \backslash\{x\})^{s \prime}$.

Proof. Necessity: Let $x \in A^{s \prime}$. Then for every supra semi open set $G$ containing $x$, we have $(G \backslash\{x\}) \bigcap A \neq \emptyset$. Therefore $(G \backslash\{x\}) \bigcap(A \backslash\{x\}) \neq \emptyset$. Thus $x \in(A \backslash\{x\})^{s \prime}$.

Sufficiency: It follows from Proposition (2). 
Theorem 1. Let $A$ be a subset of $(X, \mu)$. Then the following results hold.

(i) $A$ is a supra semi-closed set iff $A^{s \prime} \subseteq A$.

(ii) $A \cup A^{\text {s' }}$ is a supra semi-closed set.

(iii) $\operatorname{scl}(A)=A \bigcup A^{s \prime}$.

Proof.

(i) Suppose that $A$ is a supra semi-closed set and $x \notin A$. Then $A^{c}$ is a supra semi-open set containing $x$. In this case $A^{c} \cap A=\emptyset$ leads to $x \notin A^{s \prime}$. Therefore $A^{s \prime} \subseteq A$. Conversely, let $x \in A^{c}$ and let $A^{s \prime} \subseteq A$. Then $x \notin A^{s \prime}$. Therefore there is a supra semi-open set $G_{x}$ such that $G_{x} \backslash\{x\} \bigcap A=\emptyset$. Since $x \in A^{c}$, then $G_{x} \bigcap A=\emptyset$. Now, $G_{x} \subseteq A^{c}$. Therefore $A^{c}=\bigcup\left\{G_{x}: x \in A^{c}\right\}$. Thus $A$ is supra semi-closed.

(ii) Let $x \notin\left(A \bigcup A^{s \prime}\right)$. Then $x \notin A$ and $x \notin A^{s \prime}$. Therefore there is a supra semi-open set $G$ such that

$$
G \bigcap A=\emptyset
$$

Now, for each $x \in G$, we have $x \notin A^{s \prime}$. This means that

$$
G \bigcap A^{s \prime}=\emptyset
$$

From (1) and (2), we obtain $G \bigcap\left(A \bigcup A^{s \prime}\right)=\emptyset$. This implies that $x \notin\left(A \bigcup A^{s \prime}\right)^{s \prime}$. Hence $\left(A \bigcup A^{s \prime}\right)^{s \prime} \subseteq\left(A \bigcup A^{s \prime}\right)$. By (i), $A \bigcup A^{s \prime}$ is a supra semi-closed set, as required.

(iii) Since $A \subseteq \operatorname{scl}(A)$ and $A^{s \prime} \subseteq(\operatorname{scl}(A))^{s \prime} \subseteq \operatorname{scl}(A)$, then $A \bigcup A^{s \prime} \subseteq \operatorname{scl}(A)$. Since $A \cup A^{s \prime}$ is a supra semi-closed set containing $A$ and $\operatorname{scl}(A)$ is the smallest supra semi-closed set containing $A$, then $\operatorname{scl}(A) \subseteq A \bigcup A^{s \prime}$. Therefore $\operatorname{scl}(A)=A \bigcup A^{s \prime}$.

Corollary 2. If $A$ is a supra semi-closed subset of $(X, \mu)$, then $A^{s \prime},\left(A^{s \prime}\right)^{s \prime},\left(\left(A^{s \prime}\right)^{s \prime}\right)^{s \prime}, \ldots$ are supra semi-closed sets.

Definition 11. A map $g:(X, \mu) \rightarrow(Y, \nu)$ is said to be:

(i) supra semi $\imath^{\star}$-continuous if $g^{-1}(H)$ is a supra semi-open set in $X$ for every supra semi-open set in $Y$.

(ii) supra semi $\imath^{\star}$-open (resp. supra semi $\imath^{\star}$-closed) if $g(H)$ is a supra semi-open (resp. supra semi-closed) set in $Y$ for every supra semi-open (resp. supra semi-closed) set in $X$.

(iii) supra semi $i^{\star}$-homeomorphism if it is bijective, supra semi $i^{\star}$-continuous and supra semi $i^{\star}$-open.

Theorem 2. If $g:(X, \mu) \rightarrow(Y, \nu)$ is a supra semi $\imath^{\star}$-homeomorphism map, then $g\left(A^{s \prime}\right)=$ $(g(A))^{s \prime}$ for each $A \subseteq X$. 
Proof. Let $a \notin(g(A))^{s \prime}$. Then there is a supra semi-open set $H$ containing $a$ such that $(H \backslash\{a\}) \bigcap g(A)=\emptyset$. So $g^{-1}[(H \backslash\{a\}) \bigcap g(A)]=g^{-1}(\emptyset)$. This implies that $\left(g^{-1}(H) \backslash\right.$ $\left.g^{-1}(a)\right) \bigcap A=\emptyset$. Thus $g^{-1}(a) \notin A^{s \prime}$. Since $g$ is bijective, then $a \notin g\left(A^{s \prime}\right)$. Therefore $g\left(A^{s \prime}\right) \subseteq(g(A))^{s \prime}$. By reversing the preceding steps, we find that $(g(A))^{s \prime} \subseteq g\left(A^{s \prime}\right)$. Hence, the proof is complete.

Definition 12. For a nonempty set $X$, a sub collection $\Lambda$ of $2^{X}$ is said to have the difference property provided that $G \in \Lambda$ implies that $G \backslash\{x\} \in \Lambda$.

The following two examples illustrate the existence and uniqueness of the difference property.

Example 2. Let $\mu=\{\emptyset, G \subseteq \mathcal{N}: G$ is infinite $\}$ be a supra topology on the set of natural numbers $\mathcal{N}$. It is clear that the infinity of $G$ implies the infinity of $G \backslash\{x\}$. That is, $G \in \mu$ implies $G \backslash\{x\} \in \mu$. Then $(\mathcal{N}, \mu)$ has the difference property. Also, it can be seen that the collection of supra semi-open subsets of $(\mathcal{N}, \mu)$ coincides with the collection of supra open sets. Hence, $(\mathcal{N}, \mu)$ has the difference property for the collection of supra semi-open sets.

Example 3. Let $\mu=\{\emptyset, G \subseteq \mathcal{N}: G$ such that $\{1,2\} \subseteq G$ or $\{2,3\} \subseteq G\}$ be a supra topology on the set of natural numbers $\mathcal{N}$. Then $\{1,2\} \in \mu$, but $\{1,2\} \backslash\{2\}=\{1\} \notin \mu$. Therefore $(X, \mu)$ does not have the difference property.

Theorem 3. If $(X, \mu)$ has the difference property for the collection of supra semi-open sets, then the following properties hold for $A \subseteq X$.

(i) $\left(A^{s \prime}\right)^{s \prime} \subseteq A^{s \prime}$.

(ii) $\operatorname{scl}\left(A^{s \prime}\right)=A^{s \prime}=(\operatorname{scl}(A))^{s \prime}$.

(iii) $A^{s \prime}=\emptyset$ if $A$ is finite.

Proof.

(i) Let $x \notin A^{s \prime}$. Then there is a supra semi-open set $G$ containing $x$ such that $G \backslash$ $\{x\} \cap A=\emptyset$. Since $(X, \mu)$ has the difference property for the collection of supra semi-open sets, then $G \backslash\{x\}$ is a supra semi-open set. Therefore $G \backslash\{x\} \cap A^{s \prime}=\emptyset$. Since $x \notin A^{s \prime}$, then $G \bigcap\left(A^{s \prime}\right)^{s \prime}=\emptyset$. Thus $x \notin\left(A^{s \prime}\right)^{s \prime}$. Hence, $\left(A^{s \prime}\right)^{s \prime} \subseteq A^{s \prime}$.

(ii) Since $\left(A^{s \prime}\right)^{s \prime} \subseteq A^{s \prime}$, then it follows from Theorem (1) that $A^{s \prime}$ is a supra semi-closed set. Therefore

$$
\operatorname{scl}\left(A^{s \prime}\right)=A^{s \prime}
$$

Also, $(A)^{s \prime} \subseteq(\operatorname{scl}(A))^{s \prime}$ because $A \subseteq \operatorname{scl}(A)$. On the other hand, let $x \notin(A)^{s \prime}$. Then it follows from 1 above that $G \backslash\{x\} \cap A=\emptyset$ and $G \backslash\{x\} \cap A^{s \prime}=\emptyset$. This means that $G \backslash\{x\} \bigcap \operatorname{scl}(A)=\emptyset$. Therefore $x \notin(\operatorname{scl}(A))^{s \prime}$. Thus $(\operatorname{scl}(A))^{s \prime} \subseteq(A)^{s \prime}$. Hence

$$
(\operatorname{scl}(A))^{s \prime}=(A)^{s \prime}
$$

From (3) and (4), the desired result is proved. 
(iii) Let $A$ be a finite subset of $X$. Suppose that there exists an element $x \in X$ such that $x \in A^{s \prime}$. Then for every supra semi-open set $G$ containing $x$, we have $G \backslash\{x\} \cap A \neq \emptyset$. Therefore for every $y \in A$ such that $y \neq x$, we have $G \backslash\{x, y\}$ is a supra semi-open set. Thus $G \backslash[A \bigcup\{x\}]$ is a supra semi-open set such that $G \backslash[A \bigcup\{x\}] \cap A=\emptyset$. This implies that $x \notin A^{s \prime}$. But this is a contradiction. Hence, it must be that $A^{s \prime}=\emptyset$.

We explain that the three properties mentioned in the above theorem need not be true if $(X, \mu)$ does not have the difference property for the collection of supra semi-open sets. Let $A=\{1,3\}$ be a subset of supra topological space given in Example (3). Then $A^{s \prime}=\mathcal{N} \backslash\{1,3\},\left(A^{s \prime}\right)^{s \prime}=\mathcal{N}$ and $\operatorname{scl}\left(A^{s \prime}\right)=\mathcal{N}$. This leads to the following three properties.

(i) $\left(A^{s \prime}\right)^{s \prime} \nsubseteq A^{s \prime}$.

(ii) $\operatorname{scl}\left(A^{s \prime}\right) \neq A^{s \prime}$.

(iii) $A^{s \prime} \neq \emptyset$ in spite of $A$ is finite.

\section{Separation axioms with respect to supra semi-open sets}

In this section, we utilize supra semi-open sets to introduce the concepts of supra semi regular, supra semi normal and $S s T_{i}$-spaces $(i=0,1,2,3,4)$. We give some characterizations for each one of them and elucidate the relationships among themselves as well as with $S T_{i}$-space.

Definition 13. A supra topological space $(X, \mu)$ is said to be:

(i) $S s T_{0}$ if for every $a \neq b \in X$, there exists a supra semi-open set containing only one of them.

(ii) $S s T_{1}$ if for every $a \neq b \in X$, there exist two supra semi-open sets one of them contains $a$ but not $b$ and the other contains $b$ but not $a$.

(iii) supra semi Hausdorff (or $S s T_{2}$ ) if for every $a \neq b \in X$, there exist two disjoint supra semi-open sets $U$ and $V$ containing $a$ and $b$, respectively.

(iv) supra semi regular if for every supra semi-closed set $F$ and each a $\notin F$, there exist disjoint supra semi-open sets $U$ and $V$ containing $F$ and $a$, respectively.

(v) supra semi normal if for every disjoint supra semi-closed sets $F$ and $H$, there exist disjoint supra semi-open sets $U$ and $V$ containing $F$ and $H$, respectively.

(vi) $S s T_{3}$ (resp. $S s T_{4}$ ) if it is both supra semi regular (resp. supra semi normal) and $S s T_{1}$.

Theorem 4. The following three statements are equivalent:

(i) $(X, \mu)$ is an $S s T_{0}$-space; 
(ii) $\operatorname{scl}(\{a\}) \neq \operatorname{scl}(\{b\})$ for each $a \neq b \in X$;

(iii) For each $a \in X$, we have $\{a\}^{s \prime}$ is a union of supra semi-closed sets.

Proof. $1 \rightarrow 2$ : For each $a \neq b \in X$, there exists a supra semi-open set $G$ containing $a$ but not $b$, or containing $b$ but not $a$. Say $a \in G$ and $b \notin G$. Then $a \notin \operatorname{scl}(\{b\})$ because $G$ is a supra semi-open set containing $a$ such that $G \bigcap\{b\}=\emptyset$. Since $a \in \operatorname{scl}(\{a\})$, then $\operatorname{scl}(\{a\}) \neq \operatorname{scl}(\{b\})$.

$2 \rightarrow 3$ : Let $b \in\{a\}^{s \prime}$. Then $b \neq a$ and $b \in\{a\} \bigcup\{a\}^{s \prime}=\operatorname{scl}(\{a\})$. Therefore $\operatorname{scl}(b) \subseteq \operatorname{scl}(\{a\})$. Thus $b \in \operatorname{scl}(b) \subseteq\{a\}^{s \prime}$. Hence, $\{a\}^{s^{\prime}}=\bigcup\left\{\operatorname{scl}(b)\right.$ : for each $\left.b \in\{a\}^{s \prime}\right\}$.

$3 \rightarrow 1$ : Let $a \neq b$. Then we have two cases:

(i) Either $b \in\{a\}^{s \prime}$. Then there is a supra semi-closed set $F$ such that $b \in F \subseteq\{a\}^{s \prime}$. Since $a \notin\{a\}^{s \prime}$, then $a \notin F$. Therefore $F^{c}$ is a supra semi-open set containing $a$ such that $b \notin F^{c}$.

(ii) Or $b \notin\{a\}^{s \prime}$. Then there is a supra semi-open set $G$ containing $b$ such that $a \notin G$.

In the both cases above, we infer that $(X, \mu)$ is an $S s T_{0}$-space.

Corollary 3. An $S s T_{0}$-space $(X, \mu)$ contains at most a supra semi dense singleton set $(\{a\}$ is a supra semi dense set if $\operatorname{scl}\{a\}=X)$.

Proof. Let $(X, \mu)$ be an $S s T_{0}$-space. Suppose that there are two distinct singleton set $\{a\}$ and $\{b\}$ such that $\operatorname{scl}(\{a\})=\operatorname{scl}(\{b\})=X$. Then $(X, \mu)$ is not an $S s T_{0}$-space, a contradiction. Hence, $(X, \mu)$ contains at most a supra semi dense singleton set.

Theorem 5. The following four statements are equivalent:

(i) $(X, \mu)$ is an $S s T_{1}$-space;

(ii) Every singleton subset of $(X, \mu)$ is supra semi-closed;

(iii) The intersection of all supra semi-open sets containing a set $A$ is exactly A;

(iv) $\{a\}^{s \prime}=\emptyset$ for each $a \in X$.

Proof. $1 \rightarrow 2$ : Consider $(X, \mu)$ is an $S s T_{1}$-space and let $\{a\} \subseteq X$. For all $b \in X$ such that $a \neq b$, there exists a supra semi-open set $G$ containing $b$ such that $G \bigcap\{a\}=\emptyset$. Then $b \notin \operatorname{scl}(\{a\})$. Therefore, $\operatorname{scl}(\{a\})=\{a\}$. Thus, $\{a\}$ is a supra semi-closed set.

$2 \rightarrow 3$ : Let $A$ be a subset of $(X, \mu)$. Then for each $a \in A^{c}$, we have $\{a\}^{c}$ is a supra semi-open set containing $A$. Now, $A \subseteq\{G: G$ is a supra semi-open set containing $A\} \subseteq\left\{\{a\}^{c}: a \in A^{c}\right\} \subseteq A$. Thus, $A=\{G: G$ is a supra semi-open set containing $A\}$, as required.

$3 \rightarrow 4$ : Suppose that there exists $a \in X$ such that $\{a\}^{s \prime} \neq \emptyset$. Then there exists $b \neq a$ such that $b \in\{a\}^{s \prime}$. Therefore $G \backslash\{b\} \bigcap\{a\} \neq \emptyset$ for every supra semi-open set $G$ containing $b$. This implies that any supra semi-open set containing $b$ contains $a$ as well. Thus the 
intersection of all supra semi-open sets containing $b$ is not equal $\{b\}$. But this contradicts 3. Hence, it must be $\{a\}^{s \prime}=\emptyset$.

$4 \rightarrow 1$ : Let $a \neq b$. Since $\{a\}^{s \prime}=\emptyset$ and $\{b\}^{s \prime}=\emptyset$, then $\{a\}$ and $\{b\}$ are supra semiclosed sets. Therefore $\{a\}^{c}$ and $\{b\}^{c}$ are supra semi-open sets containing $\{b\}$ and $\{a\}$, respectively. Thus, $(X, \mu)$ is an $S s T_{1}$-space.

Proposition 4. Every $(X, \mu)$ satisfying the difference property for the collection of supra semi-open sets is an $S s T_{1}$-space.

Proof. Let $a \neq b \in X$. Since $X$ is a supra semi-open set and $(X, \mu)$ satisfies the difference property for the collection of supra semi-open sets, then $X \backslash\{a\}$ and $X \backslash\{b\}$ are supra semi-open sets containing $b$ and $a$, respectively, such that $a \notin X \backslash\{a\}$ and $b \notin X \backslash\{b\}$. Hence, $(X, \mu)$ is an $S s T_{1}$-space.

We show by the following example that the converse of the above proposition is not always true.

Example 4. Let $\mu=\left\{\emptyset, G \subseteq \mathcal{N}: 1 \in G\right.$, or $1 \notin G$ and $G^{c}$ is finite $\}$ be a supra topology on the natural numbers set $\mathcal{N}$. Note that $(\mathcal{N}, \mu)$ is not a topological space. Then the collection of all supra semi-open subsets of $(\mathcal{N}, \mu)$ coincides with the collection of supra open sets. It is clear that $(\mathcal{N}, \mu)$ is not an $S s T_{1}$-space. On the other hand, $(\mathcal{N}, \mu)$ does not satisfy the difference property for the collection of supra semi-open sets because $\{1,2,3\}$ is a supra semi-open set, but $\{1,2,3\} \backslash\{1\}$ is not supra semi-open.

We need the following definition to obtain the equivalence between $S s T_{0}$ and $S s T_{1}$.

Definition 14. $(X, \mu)$ is is called a supra semi symmetric space if a $\in \operatorname{scl}\{b\}$ implies that $b \in \operatorname{scl}\{a\}$ for $a \neq b \in X$.

Theorem 6. Let $(X, \mu)$ be a supra semi symmetric space. Then it is $S s T_{1}$ iff it is $S s T_{0}$.

Proof. The necessary condition is obvious.

To prove the sufficient condition, let $a \neq b$. Then there exist a supra semi-open set $G$ containing only one of them. Say, $a \in G$ and $b \notin G$. Therefore $a \notin s c l\{b\}$. By the supra semi symmetry of $(X, \mu)$, we have $b \notin \operatorname{scl}\{a\}$. Thus $(\operatorname{scl}\{a\})^{c}$ is a supra semi-open set containing $b$. Hence, $(X, \mu)$ is $S s T_{1}$.

Theorem 7. The following three statements are equivalent:

(i) $(X, \mu)$ is an $S s T_{2}$-space;

(ii) $\{a\}=\bigcap\left\{F_{i}: F_{i}\right.$ is a supra semi-closed neighborhood of a $\}$ for each $a \in X$;

(iii) The diagonal $\triangle=\{(a, a): a \in X\}$ is supra semi-closed in the product supra space $X \times X$. 
Proof. $1 \rightarrow 2$ : Consider $(X, \mu)$ is an $S s T_{2}$-space. Then for $a \neq b$, there exist two disjoint supra semi-open sets $G_{i}$ and $H_{i}$ such that $a \in G_{i}$ and $b \in H_{i}$. Obviously, $G_{i} \subseteq H_{i}^{c}$. Therefore $a \in \operatorname{scl}\left(G_{i}\right) \subseteq H_{i}^{c}=F_{i}$. Thus, $F_{i}$ is a supra semi-closed neighborhood of $a$ such that $b \notin F_{i}$. Hence, $\{a\}=\bigcap\left\{F_{i}: F_{i}\right.$ is a supra semi-closed neighborhood of $\left.a\right\}$.

$2 \rightarrow 1$ : To prove that $(X, \mu)$ is an $S s T_{2}$-space, let $a \neq b$. Since $\{a\}=\bigcap\left\{F_{i}: F_{i}\right.$ is a supra semi-closed neighborhood of $a\}$, then there exists a supra semi-closed neighborhood $F_{i_{0}}$ of $a$ such that $b \notin F_{i_{0}}$. Therefore there exists a supra semi-open set $G$ containing $a$ such that $a \in \operatorname{scl}(G) \subseteq F_{i_{0}}$. It is clear that $(\operatorname{scl}(G))^{c}$ is a supra semi-open set containing $b$ and $G \bigcap(\operatorname{scl}(G))^{c}=\emptyset$. Hence, $(X, \mu)$ is an $S s T_{2}$-space.

$1 \rightarrow 3$ : Suppose that $(X, \mu)$ is $S s T_{2}$ and let $(a, b) \in X \times X-\triangle$. Then $a \neq b$. Therefore there exist two disjoint supra semi-open sets $G$ and $H$ containing $a$ and $b$, respectively. Thus, $(a, b) \in G \times H \subseteq X \times X-\triangle$, proving that $X \times X-\triangle$ is a supra semi neighbourhood of any of its points. Hence, $\triangle$ is supra semi-closed.

$3 \rightarrow 1$ : Suppose that $\triangle$ is a supra semi-closed subset of $X \times X$ and let $a \neq b \in X$. Then $X \times X-\triangle$ is a supra semi-open set containing $(a, b)$. Therefore there exist two supra semi-open subsets $G$ and $H$ of $(X, \mu)$ such that $(a, b) \in G \times H \subseteq X \times X-\triangle$. This implies that $G$ and $H$ are two disjoint supra semi-open sets containing $a$ and $b$, respectively. Hence, $(X, \mu)$ is $S s T_{2}$.

Theorem 8. The the following three statements are equivalent:

(i) $(X, \mu)$ is supra semi regular;

(ii) For each supra semi-open subset $U$ of $(X, \mu)$ containing a, there exists a supra semiopen subset $V$ of $(X, \mu)$ such that $a \in V \subseteq \operatorname{scl}(V) \subseteq U$;

(iii) Every supra semi-open subset $U$ of $(X, \mu)$ can be represented as follows: $U=\bigcup\{H$ : $H$ is a supra semi-open subset of $(X, \mu)$ and $\operatorname{scl}(H) \subseteq U\}$.

Proof. $1 \rightarrow 2$ : Let $(X, \mu)$ be a supra semi regular space and $U$ be a supra semi-open set such that $a \in U$. Then there exist disjoint supra semi-open sets $V$ and $W$ containing $a$ and $U^{c}$, respectively. Therefore $a \in V \subseteq W^{c} \subseteq U$. Thus $a \in V \subseteq \operatorname{scl}(V) \subseteq U$.

$2 \rightarrow 3$ : Suppose that $U$ is a supra semi-open set. By hypothesise, for each $a \in U$, there exists a supra semi-open set $H$ such that $a \in H \subseteq \operatorname{scl}(H) \subseteq U$. Then $U=\bigcup\{H: H$ is supra semi-open and $\operatorname{scl}(H) \subseteq U\}$.

$3 \rightarrow 1$ : Let $F$ be a supra semi-closed set such that $a \notin F$. Then $F^{c}=\bigcup\{H: H$ is supra semi-open and $\left.\operatorname{scl}(H) \subseteq F^{c}\right\}$. Since $a \in F^{c}$, then there exists a supra semi-open set $H_{a}$ containing $a$ such that $\operatorname{scl}\left(H_{a}\right) \subseteq F^{c}$. Take $V=\left(\operatorname{scl}\left(H_{a}\right)\right)^{c}$. Then $V$ is a supra semi-open set containing $F$ and $V \cap H_{a}=\emptyset$. This completes the proof.

Theorem 9. Consider $(X, \mu)$ is a supra semi regular space. Then the following concepts are equivalent:

(i) $(X, \mu)$ is an $S s T_{2}$-space; 
(ii) $(X, \mu)$ is an $S s T_{1}$-space;

(iii) $(X, \mu)$ is an $S s T_{0}$-space.

Proof. The implications $1 \rightarrow 2 \rightarrow 3$ are obvious.

$3 \rightarrow 1$ : Let $a, b \in X$ such that $a \neq b$. Since $(X, \mu)$ is an $S s T_{0}$-space, then from Theorem (4), we get $\operatorname{scl}\{a\} \neq \operatorname{scl}\{b\}$. Therefore $a \notin \operatorname{scl}\{b\}$ or $b \notin \operatorname{scl}\{a\}$. Say, $a \notin \operatorname{scl}\{b\}$. Since $(X, \mu)$ is supra semi regular, then there exist disjoint supra semi-open sets $G$ and $H$ containing $a$ and $\operatorname{scl}\{b\}$, respectively. Thus $(X, \mu)$ is an $S s T_{2}$-space.

Theorem 10. The following statements are equivalent:

(i) $(X, \mu)$ is supra semi normal;

(ii) For each supra semi-closed set $F$ and each supra semi-open set $U$ containing $F$, there exists a supra semi-open set $V$ such that $F \subseteq V \subseteq \operatorname{scl}(V) \subseteq U$;

(iii) For every supra semi-open sets $U$ and $V$ such that $U \bigcup V=X$, there are two supra semi-closed sets $F$ and $H$ contained in $U$ and $V$, respectively, such that $F \cup H=X$.

Proof. $1 \rightarrow 2$ : Consider $(X, \mu)$ is supra semi normal and $F$ is a supra semi-closed subset of a supra semi-open set $U$. Then $U^{c}$ and $F$ are disjoint supra semi-closed sets. Therefor there exist two disjoint supra semi-open sets $W$ and $V$ containing $U^{c}$ and $F$, respectively. Thus $F \subseteq V \subseteq W^{c}=\operatorname{scl}\left(W^{c}\right) \subseteq U$. Hence, $F \subseteq V \subseteq \operatorname{scl}(V) \subseteq U$.

$2 \rightarrow 3$ : Consider $U$ and $V$ are supra semi-open sets such that $U \bigcup V=X$. Then $U^{c}$ is a supra semi-closed sets such that $U^{c} \subseteq V$. By 2, there is a supra semi-open set $G$ such that $U^{c} \subseteq G \subseteq \operatorname{scl}(G) \subseteq V$. Thus $G^{c} \subseteq U$ and $\operatorname{scl}(G) \subseteq V$ are supra semi-closed sets such that $G^{c} \bigcup \operatorname{scl}(G)=X$.

$3 \rightarrow 1$ : Consider $F$ and $H$ are disjoint supra semi-closed sets. Since $F^{c}$ and $H^{c}$ are supra open sets such that $F^{c} \cup H^{c}=X$, then there are two supra semi-closed sets $M$ and $N$ such that $M \subseteq F^{c}, N \subseteq H^{c}$ and $M \bigcup N=X$. Thus $M^{c}$ and $N^{c}$ are two disjoint supra semi-open sets containing $F$ and $H$, respectively. Hence, $(X, \mu)$ is supra semi normal.

Now, we show the implications of these separation axioms among themselves as well as with $S T_{i}$-space.

It should be noted that the concepts of $S T_{i}$-space which were defined by replacing 'supra semi-open' by 'supra open' in Definition (13), see, [2, 21].

Theorem 11. Every $S s T_{i}$-space is $S s T_{i-1}$ for $i=1,2,3,4$.

Converse of this theorem is not necessary true as it is seen in the following examples.

Example 5. Let $\mu=\{\emptyset, X,\{1\}\}$ be a supra topology on $X=\{1,2,3\}$. Then the collection of all supra semi-open subsets of $(X, \mu)$ is $\{\emptyset, X,\{1\},\{1,2\},\{1,3\}\}$. Therefore $(X, \mu)$ is not an $S s T_{1}$-space, because $1 \neq 2$ and every supra semi-open set containing 2 contains 1 as well. On the other hand, it can be checked that $(X, \mu)$ is $S s T_{0}$. 
Example 6. Let $\mu=\{\emptyset, X,\{1,2\},\{1,3\},\{2,3\},\{1,2,3\}\}$ be a supra topology on $X=$ $\{1,2,3,4\}$. Then the collection of all supra semi-open subsets of $(X, \mu)$ is $\{\emptyset, X,\{1,2\},\{1,3\}$, $\{2,3\},\{1,2,3\},\{1,2,4\},\{1,3,4\},\{2,3,4\}\}$. Therefore $(X, \mu)$ is not an $S s T_{2}$-space, because $3 \neq 4$ and there do not exist disjoint supra semi-open sets such that one of them contains 3 and the other contains 4 . On the other hand, it can be checked that $(X, \mu)$ is $S s T_{1}$.

Example 7. Let $\mu=\{\emptyset, X,\{1,2\},\{3,4\},\{1,3\},\{2,4\},\{2,3\},\{1,2,3\},\{1,2,4\},\{1,3,4\}$, $\{2,3,4\}\}$ be a supra topology on $X=\{1,2,3,4\}$. In $(X, \mu)$, every set is supra open iff it is supra semi-open. Now, $\{1,4\}$ is a supra semi-closed set and $2 \notin\{1,4\}$. Since there do not exist two disjoint supra semi-open sets such that one of them contains 2 and the other contains $\{1,4\}$, then $(X, \mu)$ is not supra $S s T_{3}$. On the other hand, it can be checked that $(X, \mu)$ is $S s T_{2}$.

Example 8. Assume that $(\mathcal{N}, \mu)$ is the same as in Example (4). Then $\{2 n: n \in \mathcal{N}\}$ and $\{2 n+3: n \in \mathcal{N}\}$ are disjoint supra semi-closed subsets of $(\mathcal{N}, \mu)$. Since there do not exist two disjoint supra semi-open sets such that one of them contains $\{2 n: n \in \mathcal{N}\}$ and the other contains $\{2 n+3: n \in \mathcal{N}\}$, then $(\mathcal{N}, \mu)$ is not supra semi normal. Therefore it is not $S s T_{4}$. On the other hand, it can be checked that $(\mathcal{N}, \mu)$ is $S s T_{3}$.

Theorem 12. Every $S T_{i}$-space $(X, \mu)$ is $S s T_{i}$ for $i=0,1,2$.

Proof. It follows from the fact that every supra open set is supra semi-open.

Converse of this theorem is not necessary true as it is seen in the following examples.

Example 9. Assume that $(X, \mu)$ is the same as in Example (5). Then $(X, \mu)$ is not an $S T_{0}$-space. On the other hand, the collection of all supra semi-open subsets of $(X, \mu)$ is $\{\emptyset, X,\{1\},\{1,2\},\{1,3\}\}$. Hence, $(X, \mu)$ is $S s T_{0}$.

Example 10. Let $\mu=\{\emptyset, X,\{1\},\{2\},\{1,2\}\}$ be a supra topology on $X=\{1,2,3\}$. Then $(X, \mu)$ is not an $S T_{1}$-space. On the other hand, the collection of all supra semi-open subsets of $(X, \mu)$ is $\{\emptyset, X,\{1\},\{2\},\{1,2\},\{1,3\},\{2,3\}\}$. Now, it can be checked that $(X, \mu)$ is $\mathrm{SsT}_{4}$.

We complete this section by discussing these separation axioms in terms of hereditary and topological properties and finite product space.

Definition 15. For a nonempty subset $A$ of $(X, \mu)$, the family $\mu_{A}=\{A \bigcap G: G$ is a supra semi-open subset of $(X, \mu)\}$ is called a relative semi-topology on $A$. A pair $\left(A, \mu_{A}\right)$ is called a semi-subspace of $(X, \mu)$.

One can easily prove that a semi-subspace $\left(A, \mu_{A}\right)$ of $(X, \mu)$ is a supra topological space.

Proposition 5. Let $\left(Y, \mu_{Y}\right)$ be a semi-subspace of $(X, \mu)$. A subset $H$ of $Y$ is supra semi-closed in $\left(Y, \mu_{Y}\right)$ iff there exists a supra semi-closed subset $F$ of $(X, \mu)$ such that $H=Y \cap F$. 
Proof. Necessity: Let $H$ be a supra semi-closed subset of $\left(Y, \mu_{Y}\right)$. Then there exists a supra semi-open subset $W$ of $\left(Y, \mu_{Y}\right)$ such that $H=Y \backslash W$. Now, there exists a supra semiopen subset $V$ of $(X, \mu)$ such that $W=Y \cap V$. Therefore $H=Y \backslash(Y \cap V)=Y \cap V^{c}$. By taking $F=V^{c}$, the proof of the necessary part is complete.

Sufficiency: Let $H=Y \cap F$ such that $F$ is a supra semi-closed subset of $(X, \mu)$. Then $Y \backslash H=Y \backslash(Y \cap F)=(Y \cap X) \backslash(Y \cap F)=Y \bigcap(X \backslash F)$. Since $X \backslash F$ is a supra semi-open subset of $(X, \mu)$, then $Y \backslash H$ is a supra semi-open subset of $\left(Y, \mu_{Y}\right)$. Thus $H$ is a supra semi-closed subset of $\left(Y, \mu_{Y}\right)$.

Definition 16. A property is said to be a relative semi-hereditary property if the property passes from a supra topological space to every relative semi-subspace.

Theorem 13. A property of being an $S s T_{i}$-space is a relative semi-hereditary for $i=$ $0,1,2,3$.

Proof. We shall suffice with proof of case $i=3$ which directly contains the case $i=1$. In a similar way, one can prove the cases $i=0,2$.

Suppose that $\left(A, \mu_{A}\right)$ is a relative semi-subspace of an $S s T_{3}$-space $(X, \mu)$. We first show that $\left(A, \mu_{A}\right)$ is an $S s T_{1}$-space. Let $x \neq y \in A \subseteq X$. Then there are two supra semi-open subsets $U$ and $V$ of $(X, \mu)$ containing $x$ and $y$, respectively, such that $x \notin V$ and $y \notin U$. Now, $G=A \bigcap U$ and $H=A \bigcap V$ are two supra semi-open subsets of $\left(A, \mu_{A}\right)$ containing $x$ and $y$, respectively, such that $x \notin H$ and $y \notin G$. Thus, $\left(A, \mu_{A}\right)$ is $S s T_{1}$. Second, we show that $\left(A, \mu_{A}\right)$ is supra semi regular. Let $H$ be a supra semi-closed subset of $\left(A, \mu_{A}\right)$ and $a \in A$ such that $a \notin H$. It follows from Proposition (5) that there is a supra semi-closed subset $F$ of $(X, \mu)$ such that $H=F \bigcap A$. Since $a \notin F$, then there exist disjoint supra semi-open subsets $U$ and $V$ of $(X, \mu)$ containing $F$ and $a$, respectively. Now, $M=U \cap A$ and $N=V \bigcap A$ are disjoint supra semi-open subsets of $\left(A, \mu_{A}\right)$ containing $H$ and $a$, respectively. Thus $\left(A, \mu_{A}\right)$ is supra semi regular. Hence, the proof is complete.

Proposition 6. Let $g:(X, \mu) \rightarrow(Y, \theta)$ be an injective supra semi-continuous map. If $(Y, \theta)$ is $T_{i}$, then $(X, \mu)$ is $S s T_{i}$ for $i=0,1,2$.

Proof. We only prove the proposition in the case of $i=2$ and the other cases can be made similarly.

Let $a \neq b \in X$. Then, it follows from the injectivity of $g$, that there are $x \neq y \in Y$ such that $x=f(a)$ and $y=f(b)$. Since $(Y, \theta)$ is $T_{2}$, then there are two disjoint open subsets $U$ and $V$ of $(Y, \theta)$ containing $a$ and $b$, respectively. Now, $g^{-1}(U)$ and $g^{-1}(V)$ are disjoint supra semi-open subsets of $(X, \mu)$ containing $a$ and $b$, respectively. Hence, $(X, \mu)$ is $S s T_{2}$, as required.

In a similar way, one can prove the following results.

Proposition 7. Let $g:(X, \tau) \rightarrow(Y, \nu)$ be a bijective supra semi-open map. If $(X, \tau)$ is $T_{i}$, then $(Y, \nu)$ is $S s T_{i}$ for $i=0,1,2$. 
Proposition 8. Let $g:(X, \tau) \rightarrow(Y, \nu)$ be an injective supra semi ${ }^{\star}$-continuous map. If $(X, \tau)$ is $S s T_{i}$, then $(Y, \nu)$ is $S s T_{i}$ for $i=0,1,2$.

Proposition 9. Let $g:(X, \tau) \rightarrow(Y, \nu)$ be a bijective supra semi $i^{\star}$-open map. If $(X, \tau)$ is $S s T_{i}$, then $(Y, \nu)$ is $S s T_{i}$ for $i=0,1,2$.

Proposition 10. Let $g:(X, \tau) \rightarrow(Y, \nu)$ be a supra semi $i^{\star}$-homeomorphism map. Then $(X, \tau)$ is $S s T_{i}$ iff $(Y, \nu)$ is $S s T_{i}$ for $i=0,1,2,3,4$.

Theorem 14. $A$ and $B$ are supra semi-open sets iff the product of them is supra semiopen.

Proof. Let $A$ and $B$ be two supra semi-open sets. Then $A \subseteq \operatorname{cl}(\operatorname{int}(A))$ and $B \subseteq$ $\operatorname{cl}(\operatorname{int}(B))$. So $A \times B \subseteq \operatorname{cl}(\operatorname{int}(A)) \times \operatorname{cl}(\operatorname{int}(B))=\operatorname{cl}(\operatorname{int}(A \times B))$. Thus $A \times B$ is a supra semi-open set. Conversely, let $A \times B$ be a supra semi-open set. Then $A \times B \subseteq$ $\operatorname{cl}(\operatorname{int}(A \times B))=\operatorname{cl}(\operatorname{int}(A)) \times \operatorname{cl}(\operatorname{int}(B))$. So $A \subseteq \operatorname{cl}(\operatorname{int}(A))$ and $B \subseteq \operatorname{cl}(\operatorname{int}(B))$. Hence, the proof is complete.

Theorem 15. The finite product of $S s T_{i}$-spaces is $S s T_{i}$ for $i=0,1,2$.

Proof. We shall suffice with proof of case $i=3$ which directly contains the case $i=1$. In a similar way, one can prove the cases $i=0,2$. For simplicity, the proof is given for two supra topological spaces $(X, \mu)$ and $(Y, \nu)$.

Let $(X \times Y, T)$ be the product supra space of $(X, \mu)$ and $(Y, \nu)$. Suppose that $\left(x_{1}, y_{1}\right) \neq$ $\left(x_{2}, y_{2}\right)$. Then either $x_{1} \neq x_{2}$ or $y_{1} \neq y_{2}$. Without loss of generality, suppose that $x_{1} \neq x_{2}$. Therefore there exist two disjoint supra semi-open subsets $U$ and $V$ of $(X, \mu)$ containing $x_{1}$ and $x_{2}$, respectively. It follows from Theorem (14) that $U \times Y$ and $V \times Y$ are two supra semi-open subsets of $(X \times Y, T)$ containing $\left(x_{1}, y_{1}\right)$ and $\left(x_{2}, y_{2}\right)$, respectively, such that $(U \times Y) \widetilde{\bigcap}(V \times Y)=\emptyset$. Hence, $(X \times Y, T)$ is $S s T_{2}$.

Definition 17. Let $(X, \mu)$ and $(Y, \nu)$ be two supra topological spaces and $(X \times Y, T)$ be their product supra space such that $\mathcal{C}_{1}$ and $\mathcal{C}_{2}$ are the collections of all supra semi-open subsets of $(X, \mu)$ and $(Y, \nu)$, respectively. Then $\beta=\left\{G \times H: G \in \mathcal{C}_{1}\right.$ and $\left.H \in \mathcal{C}_{2}\right\}$ defines a basis for a supra topology $\mathcal{C}$ on $X \times Y$. We called $(X \times Y, \mathcal{C})$ a semi-finite product supra space.

Lemma 1. Let $(X, \mu)$ and $(Y, \nu)$ be two supra topological spaces and $(X \times Y, \mathcal{C})$ be their semi-product supra space. If $E$ is a supra closed subset of $(X \times Y, \mathcal{C})$, then $E=$ $\bigcap_{i \in I}\left[\left(F_{i} \times Y\right) \bigcup\left(X \times H_{i}\right)\right]$, where $F_{i}$ and $H_{i}$ are supra semi-closed subsets of $(X, \mu)$ and $(Y, \nu)$, respectively.

Theorem 16. The semi-finite product of $S s T_{i}$-spaces is $S T_{i}$ for $i=0,1,2,3$.

Proof. We shall suffice with proof of case $i=3$ which directly contains the case $i=1$. In a similar way, one can prove the cases $i=0,2$. For simplicity, the proof is given for two supra topological spaces $(X, \mu)$ and $(Y, \nu)$. 
Let $(X \times Y, \mathcal{C})$ be the semi-product supra space of $(X, \mu)$ and $(Y, \nu)$. We first prove that $(X \times Y, \mathcal{C})$ is $S T_{1}$. Suppose that $\left(x_{1}, y_{1}\right) \neq\left(x_{2}, y_{2}\right)$. Then either $x_{1} \neq x_{2}$ or $y_{1} \neq$ $y_{2}$. Without loss of generality, suppose that $x_{1} \neq x_{2}$. Therefore there exist two supra semi-open subsets $U$ and $V$ of $(X, \mu)$ containing $x_{1}$ and $x_{2}$, respectively. According to Definition(17), $U \times Y$ and $V \times Y$ are two supra open subsets of $(X \times Y, \mathcal{C})$ containing $\left(x_{1}, y_{1}\right)$ and $\left(x_{2}, y_{2}\right)$ such that $\left(x_{1}, y_{1}\right) \notin V \times Y$ and $\left(x_{2}, y_{2}\right) \notin U \times Y$. Hence, $(X \times Y, \mathcal{C})$ is $S T_{1}$. Second, we prove that $(X \times Y, \mathcal{C})$ is supra regular. Suppose that $(x, y) \in X \times Y$ and $E$ is a supra closed subset of $(X \times Y, \mathcal{C})$ such that $(x, y) \notin E=\bigcap_{i \in I}\left[\left(F_{i} \times Y\right) \bigcup\left(X \times H_{i}\right)\right]$, where $F_{i}$ and $H_{i}$ are supra semi-closed subsets of $(X, \mu)$ and $(Y, \nu)$, respectively. Then there exists $j \in I$ such that $(x, y) \notin\left[\left(F_{j} \times Y\right) \bigcup\left(X \times H_{j}\right)\right]$. This means that $x \notin F_{j}$ and $y \notin H_{j}$. Since $(X, \mu)$ and $(Y, \nu)$ are supra semi regular, then there exist disjoint supra semiopen subsets $U$ and $V$ of $(X, \mu)$ containing $x$ and $F_{j}$, respectively, and there exist disjoint supra semi-open subsets $M$ and $N$ of $(Y, \nu)$ containing $y$ and $H_{j}$, respectively. Therefore $U \times M$ and $[(V \times Y) \bigcup(X \times N)]$ are two supra open subsets of $(X \times Y, \mathcal{C})$ containing $(x, y)$ and $\left[\left(F_{j} \times Y\right) \bigcup\left(X \times H_{j}\right)\right]$, respectively. Obviously, $E \subseteq\left[\left(F_{j} \times Y\right) \bigcup\left(X \times H_{j}\right)\right]$ and $(U \times M) \bigcap[(V \times Y) \bigcup(X \times N)]=\emptyset$. Thus, $(X \times Y, T)$ is supra regular. Hence, the proof is complete.

\section{Conclusion}

There are many generalizations of topological spaces which help us to picture and satisfy many topological properties under fewer conditions such as supra topology, minimal structure and generalized topology. This work is devoted to introducing and discussing the concepts of limits points of a set and separation axioms with respect to semi-open sets. We have established their main properties and provided some examples to show the obtained results. From the concrete thoughts given in this work, it can be done more investigations on the theoretical parts of these generalized ideas which is valuable by studying the following themes:

(i) Define weak types of supra semi regular and supra semi normal spaces.

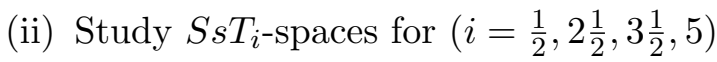

(iii) Explore the concepts introduced herein using the classes of supra $\alpha$-open sets, supra $b$-open sets and supra $\beta$-open sets.

(iv) Investigate of the possibility of applying these concepts on information system, especially, separation axioms.

\section{Conflict of interest}

The authors declare that there is no conflict of interest regarding the publication of this paper. 


\section{Acknowledgements}

The authors would like to thank the reviewers for their valuable comments which improved the presentation of this paper.

\section{References}

[1] A M Al-Odhari. On infra topological spaces. International Journal of Mathematical Archive, 6(11):179-184, 2015.

[2] T M Al-shami. Some results related to supra topological spaces. Journal of Advanced Studies in Topology, 4(7):283-294, 2016.

[3] T M Al-shami. On supra semi open sets and some applications on topological spaces. Journal of Advanced Studies in Topology, 8(2):144-153, 2017.

[4] T M Al-shami. Somewhere dense sets and $S T_{1}$-spaces. Punjab University Journal of Mathematics, 49(2):101-111, 2017.

[5] T M Al-shami. Utilizing supra $\alpha$-open sets to generate new types of supra compact and supra lindelöf spaces. Facta Universitatis, Series: Mathematics and Informatics, 32(1):151-162, 2017.

[6] T M Al-shami. Supra semi-compactness via supra topological spaces. Journal of Taibah University for Science, 12(3):338-343, 2018.

[7] T M Al-shami. Paracompactness on supra topological spaces. Journal of Linear and Topological Algebra, 9(2):121-127, 2020.

[8] T M Al-shami, B A Asaad, and M A El-Gayar. Various types of supra pre-compact and supra pre-lindelöf spaces. Missouri Journal of Mathematical Science, 32(1):1-20, 2020 .

[9] T M Al-shami and M E El-Shafei. On supra soft topological ordered spaces. Arab Journal of Basic and Applied Sciences, 26(1):433-445, 2019.

[10] T M Al-shami and M E El-Shafei. Two types of separation axioms on supra soft topological spaces. Demonstratio Mathematica, 52(1):147-165, 2019.

[11] T M Al-shami and T Noiri. More notions and mappings via somewhere dense sets. Afrika Matematika, 30(7):1011-1024, 2019.

[12] P Alexandroff. Diskrete räume. Rec. Math. [Mat. Sbornik] N.S., 2(44):501-519, 1937.

[13] Á Császár. Generalized topology, generalized continuity. Acta Mathematica Hungarica, 96:351-357, 2002.

[14] Á Császár. Weak structure. Acta Mathematica Hungarica, 131(11):193-195, 2011. 
[15] R Devi, S Sampathkumar, and M Caldas. On $\alpha$-open sets and $s \alpha$-continuous maps. General Mathematics, 16:77-84, 2008.

[16] M E El-Shafei, M Abo-Elhamayel, and T M Al-shami. On supra R-open sets and some applications on topological spaces. Journal of Progressive Research in Mathematics, 8(2):1237-1248, 2016.

[17] M E El-Shafei, A H Zakari, and T M Al-shami. Some applications of supra preopen sets. Journal of Mathematics, Volume 2020, Article ID 9634206:11 pages, 2020.

[18] S Jafari and S Tahiliani. Supra $\beta$-open sets and supra $\beta$-continuity on topological spaces. Annales Univ. Sci. Budapest., 56:1-9, 2013.

[19] A M Kozae, M Shokry, and M Zidan. Supra topologies for digital plane. AASCIT Communications, 3(1):1-10, 2016.

[20] H Maki, J Umehara, and T Noiri. Every topological space is pret $t_{\frac{1}{2}}$. Mem. Fac. Sci. Kochi. Univ. Ser. A Math., 17:33-42, 1996.

[21] A S Mashhour, A A Allam, F S Mahmoud, and F H Kheder. On supra topological spaces. Indian Journal of Pure and Applied Mathematics, 14(4):502-510, 1983.

[22] J M Mustafa. Supra b-compact and supra b-lindelof spaces. Journal of Mathematics and Applictions, 36:79-83, 2013.

[23] J M Mustafa and H A Qoqazeh. Supra d-sets and associated separation axioms. International Journal of Pure and Applied Mathematics, 80(5):657-663, 2012.

[24] O R Sayed. Supra pre-open sets and supra pre-continuous on topological spaces. Series Mathematics and Information, 20(2):79-88, 2010.

[25] O R Sayed. Supra $\beta$-connectedness on topological spaces. Proceedings of the Pakistan Academy of Sciences, 49(1):19-23, 2012.

[26] O R Sayed and T Noiri. On supra b-open sets and supra b-continuity on topological spaces. European Journal of Pure and Applied Mathematics, 3:295-302, 2010. 